\title{
Outcomes of Conservative Management of Acute Appendicitis during COVID-19 Pandemic
}

\author{
Muzna Iftikhar ${ }^{1}$, Sajida Shah², Inamullah Shah ${ }^{1}$, Jamil Ahmad Shah ${ }^{1}$ and Mahvish Faisal ${ }^{1}$ \\ ${ }^{1}$ Department of Surgery, Foundation University, Islamabad, Pakistan \\ ${ }^{2}$ Department of Radiology, Shifa International Hospital, Islamabad, Pakistan
}

\begin{abstract}
Objective: To measure the outcomes of conservative treatment of acute appendicitis.

Study Design: Observational study.

Place and Duration of Study: Fauji Foundation Hospital, Rawalpindi from April to July 2020.

Methodology: All 58 patients $(n=58)$ presenting with acute appendicitis (AA) were included. Assessment was done with Alvarado score (AS) and ultrasound. Treatment was initiated according to the algorithm corresponding with AS. Those with AS score of 4 or less were started on outpatient oral antibiotics. Patients with AS score of 5 or more were admitted for IV antibiotics. If the symptoms and signs resolved, they were sent home on oral antibiotics to complete a course of 5 days. If their condition did not improve in 72 hours or deteriorated at any time, appendectomy was done. Outcomes were recorded and analysed on SPSS.

Results: Out of 58 patients, 16 were treated with oral, while 42 with IV antibiotics. This yielded a statistically significant difference on the course of disease $(p=0.028)$. Resolution of symptoms was seen in $27.6 \%$ ( $n=16)$ with conservative management; whereas, $72.4 \%(n=42)$ patients needed a subsequent appendectomy. The difference in operative findings between patients, who had been given oral or IV antibiotics was statistically insignificant $(p=0.536)$. Diagnostic value of leukocyte count (TLC), ultrasound and AS was not found to be significant.

Conclusion: Non-operative management is successful in about a quarter of the patients of AA. There is very limited value of sonography, laboratory parameters, or AS in confirming the diagnosis of AA.
\end{abstract}

Key Words: Appendicitis, Conservative treatmen, COVID-19.

How to cite this article: Iftikhar M, Shah S, Shah I, Shah JA, Faisal M. Outcomes of Conservative Management of Acute Appendicitis during COVID-19 Pandemic. J Coll Physicians Surg Pak 2021; 31(JCPSPCR):CR50-CR54.

\section{INTRODUCTION}

COVID-19 pandemic has led to thinned out operation lists because the guidelines recommend conservative management, wherever possible, to release the burden on the healthcare system. ${ }^{1,2}$ Stout clinical judgment is essential to maintain balance in ER, directing resources to the most essential surgeries. Surgical emergencies, such as blunt force trauma orpenetrating injuries, usually require immediate or sequential operative care. ${ }^{3}$ Acute appendicitis (AA) is one of the common presentations in ER. Gold standard treatment is appendectomy via open or laparoscopic approach. ${ }^{4}$

Conservative management of AA has been advocated in literature by some authorsdespite varied results reported in previous trials. $^{2,4}$

Correspondence to: Dr. Muzna Iftikhar, Department of Surgery, Fauji Foundation Hospital, Rawalpindi, Pakistan E-mail: muz_272@hotmail.com

Received: September 07, 2020; Revised: January 18, 2021; Accepted: February 11, 2021

DOI: https://doi.org/10.29271/jcpsp.2021.JCPSPCR.CR50
Assessment for AA depends on clinical parameters with imaging and laboratory being used as adjuncts. Various scoring systems such as AS, Saint-Antoine, RIPASA, and algorithms like AIR have evolved and are being sporadically used in the diagnosis of AA. ${ }^{5}$ An AS of 4 or less has been claimed to virtually exclude appendicitis, while a score of 5 or 6 corresponds to uncomplicated AA, increasing the chance of successful conservative management by antibiotics alone. ${ }^{1}$ With a significant population of asymptomatic COVID-19 patients and a large proportion of false negative PCR tests, administering general anesthesia and performing surgery poses a considerable risk to everyone in the closed environment of an operating room. Recent studies reveal that patients with COVID-positive status to have a worse recovery than regular postoperative patients with the same disease. ${ }^{1,2}$ Therefore, COVID-19 guidelines for elective and emergency surgery recommend initial conservative management for AA in order to decrease exposure. ${ }^{1}$

Starting in April 2020, when COVID-19 patients started appearing in increasing numbers, this institution adopted protocols with a similar approach. Suspected AA was initially managed conservatively, and surgical intervention being reserved for only those judged to have perforation. This was an opportunity to assess the outcomes of non-operative manage- 
ment in AA as COVID-19 protocols adopted by the hospital mandated a conservative trial ofevery case of AA unless perforation was suspected. Patient selection in previous studies was limited to cases with uncomplicated or mild AA., Previous studies were also constrained by patient consent in choosing between operative and non-operative management. ${ }^{6}$

The objective of this study was to measure outcomes of conservative treatment of acute appendicitis in a tertiary care setting.

\section{METHODOLOGY}

This is a prospective observational study carried out in Surgical Department of Fauji Foundation Hospital, Rawalpindi from April to July 2020, afterfulfilling ethical considerations of Institutional Review Board. All patients, regardless of age and gender, presenting with symptoms of right iliac fossa pain and suspicion of $A A$, were included in this study. Patients with suspected perforation, who were operated urgently, were excluded. Patients were counselled and consents obtained before proceeding.

Assessment was done by using $\mathrm{AS}^{8,9}$ and trans-abdominal ultrasound of abdomen and pelvis. Ultrasound criteria suggestive of AA included probe tenderness in right iliac fossa, diameter of appendix $1 \mathrm{~cm}$ or more, and an aperistaltic gut loop in the vicinity of appendix.

If perforation was suspected at presentation, urgent surgery was performed with full precautions, according to COVID-19 protocol. Patients with mild symptoms and AS $\leq 4$ were treated on outpatient basis with oral antibiotics and followed up after three days or earlier, if symptoms worsened. Patients with AS $\geq 5$ were admitted, oral intake was stopped, intravenous fluid infusion started, and IV antibiotics were administered. Antibiotic regimen for inpatient care was intravenous cefuroxime $1500 \mathrm{mg} 8$ hourly and metronidazole $500 \mathrm{mg} 8$ hourly. COVID-19 screening test was obtained and patients were reassessed periodically. If signs and symptoms of AA resolved completely on this regimen, patient was discharged. Oral antibiotics were continued to complete a five-day course. Outpatient treatment comprised oral ciprofloxacin 500mg 12-hourly and metronidazole $400 \mathrm{mg}$ thrice daily. If symptoms failed to resolve or worsened at any time during 72 hours after admission, appendectomy was performed.

Primary outcome measures were effect of non-operative treatment on course of the disease, need for index appendectomy, operative findings, and difference in operative findings in patients initially treated with oral or IV antibiotics. Secondary outcomes included effectiveness of TLC, ultrasound and AS in diagnosis of AA. Data was recorded and analysed on SPSS version 23. Percentages and counts were used to describe qualitative variables. Chi-square likelihood ratio was used to find out significance of difference between variables. P-value of $<0.05$ was considered significant.

\section{RESULTS}

The total study sample comprised 58 patients. Out of these,
$79.3 \%(n=46)$ were females and $20.7 \%(n=12)$ were males. Patients 18 years of age or less were $53.4 \%(n=31), 36.2 \%$ $(n=21)$ were between 19 and 60 years; whereas $10.3 \%(n=6)$ were above 60 years of age. It was found that $69 \%(n=40)$ patients presented to this Hospital within 2 days, $20.7 \%(n=12)$ presented on day 3 or 4 , and $10.3 \%(n=6)$ presented on day 5 or later after onset of pain in the abdomen. Clinical features at the time of presentation are given in Tablel.

Table I: Frequency of clinical features.

\begin{tabular}{|l|c|c|c|c|}
\hline \multirow{2}{*}{ Clinical feature } & \multicolumn{2}{|c|}{ Yes } & \multicolumn{2}{c|}{ No } \\
\cline { 2 - 5 } & $\%$ & $\mathbf{n}$ & $\%$ & $\mathbf{n}$ \\
\hline History of pain migration & 51.7 & 30 & 48.3 & 28 \\
\hline Pain right iliac fossa & 96.6 & 56 & 3.4 & 2 \\
\hline Vomiting & 75.9 & 44 & 24.1 & 14 \\
\hline Fever & 17.2 & 10 & 82.8 & 48 \\
\hline Tenderness RIF & 94.8 & 55 & 5.2 & 3 \\
\hline
\end{tabular}

The total leucocyte count (TLC) of $39.7 \%(n=23)$ patients was within normal range $\left(4000-11000 / \mathrm{mm}^{3}\right) ; 50 \%(n=29)$ had TLC ranging between $11000-20000 / \mathrm{mm}^{3}$; and $10.3 \%(n=6)$ had TLC above $20,000 / \mathrm{mm}^{3}$. Ultrasound scan in $58.6 \%(n=34)$ was suggestive of acute appendicitis whereas it showed no abnormality in $41.4 \%(n=24)$. AS was $2-4$ in $44.8 \%(n=26) ; 5-6$ in $44.8 \%(n=26)$; and $7-8$ in $10.3 \%(n=6)$.

Conservative treatment had a statistically significant difference on the course of disease $(p=0.028)$. Association of mode of conservative treatment with course of the disease is shown in Tablell.

Patients, whose symptoms resolved with conservative treatment, were $27.6 \%(n=16)$; whereas, $72.4 \%(n=42)$ patients needed appendectomy after a trial of conservative treatment. Duration of indoor IV antibiotic treatment varied from two to four days. All patients completed five days of antibiotic treatment. Most patients, who needed appendectomy, were operated within first three days of trial with antibiotics. Peroperative findings in patients, who had to undergo appendectomy during non-operative management, are given in Table III.

Conservative treatment was offered to 58 patients, out of which 16 patients were treated with oral antibiotics, while 42 were treated with intravenous antibiotics. The difference in operative findings between patients, who had been given oral or IV antibiotics, was statistically insignificant $(p=0.536)$. Days since onset of symptoms at the time of starting treatment, made no significant difference to the course of disease $(p=0.100)$, but it made significant difference in peroperative findings $(p=0.044)$.

Difference in peroperative findings was statistically insignificant when cross-tabulated with total leukocyte count $(p=0.366)$ and preoperative sonographic findings $(p=0.505)$.

Preoperative AS of patients, who underwent surgery, was analysed with their operative findings, applying Chi-square test. The results showed significant difference between the predic- 
tive AS and actual operative findings $(p=0.001)$.

Table II: Course of disease in patients on conservative treatment.

\begin{tabular}{|c|c|c|c|c|c|}
\hline \multirow{2}{*}{$\begin{array}{l}\text { Mode of conservative } \\
\text { treatment }\end{array}$} & \multicolumn{3}{|c|}{ Course of disease } & \multirow[b]{2}{*}{ Total } & \multirow[b]{2}{*}{ p-value } \\
\hline & $\begin{array}{l}\text { Symptoms } \\
\text { resolved }\end{array}$ & $\begin{array}{l}\text { Need for index } \\
\text { appendectomy }\end{array}$ & $\begin{array}{l}\text { Recurrence leading } \\
\text { to appendectomy }\end{array}$ & & \\
\hline Oral antibiotics & $6(37.5 \%)$ & $8(50 \%)$ & $2(12.5 \%)$ & $16(100 \%)$ & \multirow{2}{*}{0.028} \\
\hline IV antibiotics & $10(23.8 \%)$ & $32(76.2 \%)$ & $0(0)$ & $42(100 \%)$ & \\
\hline Total & $16(27.6 \%)$ & $40(69 \%)$ & $2(3.4 \%)$ & $58(100 \%)$ & \\
\hline
\end{tabular}

Table III: Operative findings after trial of conservative treatment.

\begin{tabular}{|c|c|c|c|c|c|c|}
\hline \multirow{2}{*}{$\begin{array}{l}\text { Mode of conservative } \\
\text { treatment }\end{array}$} & \multicolumn{4}{|c|}{ Operative findings } & \multirow[b]{2}{*}{ Total } & \multirow[b]{2}{*}{ p-value } \\
\hline & Appendix inflamed & $\begin{array}{l}\text { Perforation / Near } \\
\text { Perforation / Mass }\end{array}$ & $\begin{array}{c}\text { Gangrene with } \\
\text { localised peritonitis }\end{array}$ & $\begin{array}{c}\text { Generalised } \\
\text { peritonitis }\end{array}$ & & \\
\hline Oral antibiotics & $2(20 \%)$ & $6(60 \%)$ & $2(20 \%)$ & $0(0)$ & $10(100 \%)$ & \multirow{3}{*}{0.536} \\
\hline IV antibiotics & $11(34.4 \%)$ & $15(46.9 \%)$ & $4(12.5 \%)$ & $2(6.2 \%)$ & $32(100 \%)$ & \\
\hline Total & $13(31 \%)$ & $21(50 \%)$ & $6(14.3 \%)$ & $2(4.7 \%)$ & $42(100 \%)$ & \\
\hline
\end{tabular}

\section{DISCUSSION}

This study was conducted to find out the outcomes of non-operative management of acute appendicitis as determined by resolution of acute appendicitis or the need to perform appendectomy. The results revealed that signs and symptoms of acute appendicitis resolved in only $27.6 \% \quad(n=16)$ patients with conservative management. An overwhelming $72.4 \%(n=42)$ patients had to be operated because either they did not improve or worsened with non-operative management. This is in conformity with a meta-analysis of 1,351 patients that reported higher treatment efficacy and low complication rate with appendectomy $(p<0.001) .{ }^{10}$ Conversely, Becker et al. reported $90 \%$ success rate of conservative treatment. ${ }^{11}$ Yang et al. reported a high rate of relapse even after resolution of symptoms with antibiotic treatment. ${ }^{12}$ All these studies, like present study, included cases of all age groups. A recent multicentre trial in the US reports $67.1 \%$ efficacy of nonoperative treatment in pediatric population with uncomplicated acute appendicitis with sonographic diameter of appendix less than $1.1 \mathrm{~cm} .{ }^{6}$ This high success rate of non-operative treatment may be attributed to selection of uncomplicated cases of appendicitis with mild symptoms and equivocal signs.

The results of this study reveal that out of 42 patients, who progressed to appendectomy during non-operative management, $76.2 \% \quad(n=32)$ were treated with IV antibiotics as indoor patients; whereas, $23.8 \%(n=10)$ were treated as outdoor patients with oral antibiotics. The difference in outcome after treatment with oral and IV antibiotics was significant $(p=0.028$, Table II); but the difference in operative findings of patients treated with these two methods was insignificant $(p=0.536$, Table (II). Broad spectrum oral antibiotics along with metronidazole are frequently used in selected cases of mild appendicitis with strict instructions on follow-up. In a pandemic scenario, where most patients were wary of getting admitted in a hospital, they were offered the choice of oral antibiotics to selected cases, based on their symptoms, clinical signs, sonographic findings and AS. Results of the first randomised controlled trial, APPAC II trial, comparing oral and intravenous antibiotics in the treatment of uncomplicated acute appendicitis confirmed on CT scan, are still awaited. ${ }^{7}$

The results of this study revealed that TLC is of limited use in diagnosis of acute appendicitis as difference in operative findings was not statistically significant with various counts $(p=0.366)$. This is in contrast to a recent South African study by Virmani et al., which reports a total count $\geq 13500 / \mathrm{mm}^{3}$ to be associated with acute appendicitis. ${ }^{13}$ Ultrasound (USG) and CT scan are frequently used to confirm clinical diagnosis of appendicitis. ${ }^{14}$ USG was carried out in all cases in this study. Probe tenderness, diameter of appendix more than $1 \mathrm{~cm}$, and non-peristaltic gut loops in the vicinity of appendix were considered to be suggestive of acute appendicitis. Results of this study revealed that USG findings were not always in conformity with peroperative findings. The difference in surgical findings between sonographically positive and negative cases was statistically insignificant $(p=0.505)$. This is in conformity to a systematic review by Benabbas et al., which concluded that an ultrasound scan not suggestive of appendicitis, does not rule out acute appendicitis. ${ }^{15}$

Various scoring systems and algorithms have evolved to make the diagnosis of acute appendicitis more objective. These include algorithms based on artificial intelligence or machine learning, ${ }^{16}$ and various scoring systems. ${ }^{17}$ Most commonly used scoring systems are RIPASA and AS with sensitivity of $94 \%$ and $69 \%$, 
respectively; ${ }^{18}$ although, some studies have found no advantage of one over the other. ${ }^{7}$ The authors used AS as an aid to supplement clinical judgment of acute appendicitis. Results of this study showed that AS is of limited value in diagnosis of appendicitis. An AS of less than 5 is reported to be $99 \%$ sensitive in excluding the diagnosis of acute appendicitis; ${ }^{17}$ but in this study, there was significant difference in the diagnosis suggested by $A S$ and the one found during surgery $(p=0.001)$. Although previous studies have reported better figures in favour of AS, ${ }^{7,17-19} 2020$ Update on World Society of Emergency Surgery Jerusalem Guidelines suggest that a score that combines clinical and ultrasound parameters, like appendicitis inflammatory response (AIR) score, may improve sensitivity and specificity for diagnosis without resorting to a CT scan. ${ }^{17}$

Literature search showed that all studies reporting nonoperative management of acute appendicitis included patients who opted for it. ${ }^{11,12,14}$ Those who opted for surgery were excluded. This was a selection pitfall as patients who consented or were included for study necessarily had a relatively mild disease in terms of symptoms and signs. During COVID-19 pandemic, most patients with acute appendicitis were managed nonoperatively. ${ }^{20}$ Peculiar circumstances leading to a change in favour of non-operative treatment protocols enabled the authors to carry out this study without selection bias. Despite a relatively small sample size, this is the strength of this study.

\section{CONCLUSION}

Non-operative management is successful only in about a quarter of the patients of simple and complicated acute appendicitis. No single criterion, including ultrasound, laboratory parameters or AS can be relied upon to confirm the diagnosis in suspected acute appendicitis.

\section{DISCLOSURE:}

No funding was obtained for this study from an individual, organization or institution. This study was not a part of a thesis or dissertation.

\section{UNDERTAKING:}

This paper is the original research work of the authors and has not been submitted for a concurrent publication, or published before in any other journal.

\section{ETHICAL APPROVAL:}

Approval was obtained from Institutional Review Board.

\section{CONFLICT OF INTEREST:}

The authors declared no conflict of interest.

\section{PATIENTS' CONSENT:}

Patients were counselled and consents obtained before proceeding.

\section{AUTHORS' CONTRIBUTION:}

MI: Conceived the research question, methodology, performed surgeries, and authored the literature.

SS: Contributed by performing ultrasounds of all patients, and revising the draft manuscript.

IS: Contributed in designing the methodology, and performed data analysis.

JAS: Contributed in data collection, performance of surgeries, literature search and reading the manuscript. MI: Contributed in data collection.

All authors have agreed on the final manuscript and are accountable for all aspects of the work.

\section{REFERENCES}

1. Moletta L, Pierobon ES, Capovilla G, Costantini M, Salvador R, Merigliano $\mathrm{S}$, et al. International guidelines and recommendations for surgery during Covid-19 pandemic: A systematic review. Int J Surg 2020; 79:180-8. doi: 10.1016/j.ijsu.2020.05.061.

2. Javanmard-Emamghissi $H$, Boyd-Carson $H$, Hollyman $M$, Doleman B, Adiamah A, Lund JN et al. The management of adult appendicitis during the COVID-19 pandemic: An interim analysis of a UK cohort study. Tech Coloproctol 2020; 1-11. http: //link.springer.com/article/10.1007\%2Fs10151-020-022 97-4.

3. Coimbra R, Edwards S, Kurihara H. European society of trauma and emergency surgery (ESTES) recommendations for trauma and emergency surgery preparation during times of COVID-19 infection. Eur J Trauma Emerg Surg 2020; 46(3):505-10. doi: 10.1007/s00068-020-01364-7

4. Salminen P, Paajanen $H$, Rautio $T$, Nordström $P$, Aarnio $\mathrm{M}$, Rantanen $\mathrm{T}$, et al. Antibiotic therapy vs. appendectomy for treatment of uncomplicated acute appendicitis: The APPAC randomised clinical trial. JAMA 2015; 313(23):2340-8. doi: 10.1001/ jama. 2015. 6154.

5. Ak R, Doğanay F, Unal Akoğlu E, Akoğlu H, Uçar AB, Kurt $E$, et al. Predictive value of scoring systems for the diagnosis of acute appendicitis in emergency department patients: Is there an accurate one? Hong Kong J Emergency Med 2019; 27(5): doi.org/10. 1177/ 1024907919840175.

6. Minneci $P C$, Hade $E M$, Lawrence $A E$, Sebastião $Y V$, Saito JM, Mak GZ, et al. Association of non-operative management using antibiotic therapy vs. laparoscopic appendectomy with treatment success and disability days in children with uncomplicated appendicitis. JAMA 2020; 324(6):581-93. doi:10.1001/jama.2020. 10888

7. Haijanen J, Sippola S, Grönroos J, Rautio T, Nordström $\mathrm{P}$, Rantanen $\mathrm{T}$, et al. Optimising the antibiotic treatment of uncomplicated acute appendicitis: A 
protocol for a multicenter randomised clinical trial (APPAC II trial). BMC Surg 2018; 18(1):117. DOI: 10.1186/ s12893-018-0451-y.

8. Khan S, Usama M, Basir Y, Muhammad S, Jawad M, Khan $\mathrm{T}$, et al. Evaluation of modified Alvarado, Ripasa and Lintula scoring system as diagnostic tools for acute appendicitis. J Ayub Med Coll Abbottabad 2020; 32(1):46-50.

9. Frountzas M, Stergios K, Kopsini D, Schizas D, Kontzoglou K, Toutouzas K. Alvarado or RIPASA score for diagnosis of acute appendicitis? A meta-analysis of randomized trials. Int J Surg 2018; 56:307-14. doi: 10.1016/j.ijsu.2018.07.003.

10. Podda M, Cillara N, Di Saverio S, Lai A, Feroci F, Luridiana $G$, et al. Antibiotics-first strategy for uncomplicated acute appendicitis in adults is associated with increased rates of peritonitis at surgery. A systematic review with meta-analysis of randomized controlled trials comparing appendectomy and nonoperative management with antibiotics. Surgeon 2017; 15(5): 303-14. doi: 10.1016/j.surge.2017. 02.001.

11. Becker P, Fichtner-Feigl S, Schilling D. Clinical management of appendicitis. Visc Med 2018; 34(6): 453-58. doi.org/10.1159/000494883.

12. Yang Z, Sun F, Ai S, Wang J, Guan W, Liu S. Metaanalysis of studies comparing conservative treatment with antibiotics and appendectomy for acute appendicitis in the adult. BMC Surg 2019; 19(1):110. doi: 10.1186/ s12893-019-0578-5.

13. Virmani S, Prabhu PS, Sundeep PT, Kumar V. Role of laboratory markers in predicting severity of acute appendicitis. Afr J Paediatr Surg 2018; 15(1):1-4.

14. Snyder MJ, Guthrie M, Cagle S. Acute appendicitis: Efficient diagnosis and management. Am Fam Physician 2018; 98(1):25-33.

15. Benabbas R, Hanna M, Shah J, Sinert R. Diagnostic accuracy of history, physical examination, laboratory tests, and point-of-care ultrasound for pediatric acute appendicitis in the emergency department: A systematic review and meta-analysis. Acad Emerg Med 2017; 24(5):523-51. doi: 10.1111/acem.13181.

16. Reismann J, Romualdi A, Kiss N, Minderjahn M, Kallarackal J, Schad $M$, et al. Diagnosis and classification of pediatric acute appendicitis by artificial intelligence methods: An investigatorindependent approach. PLoS One 2019; 14(9):e022 2030. doi: 10.1371/journal.pone.0222030.

17. Di Saverio S, Podda $M$, De Simone $B$, Ceresoli $M$, Augustin G, Gori A, et al. Diagnosis and treatment of acute appendicitis: 2020 update of the WSES Jerusalem guidelines. World J EmergSurg 2020; 15(27): 306-14. doi: 10.1186/s13017-020-00306-3.

18. Noor S, Wahab A, Afridi G, Ullah K. Comparing Ripasa score and Alvarado score in an accurate diagnosis of acute appendicitis. J Ayub Med Coll Abbottabad 2020; 32(1):38-41.

19. Kundiona I, Chihaka OB, Muguti GI. Negative appendicectomy: Evaluation of ultrasonography and Alvarado score. Cent Afr J Med 2015; 61(9-12):66-73.

20. COVID Surg Collaborative. Global guidance for surgical care during the COVID-19 pandemic. Br J Surg 2020; 15:10. www.ncbi.nlm.nih.gov/pmc/articles/ 